\title{
ARRIBOFLAVINOSE CLINICA EM PRÉ-ESCOLARES, FILHOS DE MIGRANTES EM TRÂNSITO PELA CENTRAL DE TRIAGEM E ENCAMINHAMENTO, NA CAPITAL DO ESTADO DE SÃO PAULO, BRASIL.
}

Donald Wilson *

Maria José Roncada*

Olderigo Berretta Netto **

Adamo Lui Netto $* * *$

Aldonia C. Kalil ****

Maria de Fátima Nunes *

RSPUB $9 / 423$

Wilson, D. et al. Arriboflavinose clinica em pré-escolares, filhos de migrantes em trânsito pela Central de Triagem e Encaminhamento, na Capital do Estado de São Paulo, Brasil. Rev. Saúde públ., S. Paulo, 12: $351-5,1978$.

RESUMO: Foi realizado um levantamento clinico entre 109 pré-escolares, filhos de migrantes em trânsito pela Central de Triagem e Encaminhamento (CETREN), tendo-se constatado altissima prevalência de arriboflavinose. A única medida factivel foi a administraçâo de riboflavina a todas as crianças examinadas.

UNITERMos: Arriboflavinose, pré-escolares. Riboflavina.

\section{N T RODU C A O}

Desde 1969, vem chamando nossa atenção a alta prevalência de sinais clínicos atribuiveis à arriboflavinose, cujo coeficiente era da ordem de $30 \%$ das pessoas examinadas em várias localidades do Estado de São Paulo', considerando-se a presença de pelo menos um sinal clinico.
Recentemente, iniciamos uma pesquisa sobre cegueira e hipovitaminose A, em préescolares filhos de migrantes em trânsito pela Central de Triagem e Encaminhamento (CETREN), órgão da Secretaria da Promoção Social do Estado de São Paulo, Capital. Neste trabalho, em que se utili-

* Do Departamento de Nutrição da Faculdade de Saúde Pública da Universidade de São Paulo - Av. Dr. Arnaldo, 715 - São Paulo, SP - Brasil.

* Do Hospital Infantil da Cruz Vermelha de São Paulo - Av. Moreira Guimarães, 699 04074 - São Paulo. SP - Brasil.

** Do Departamento de Oftalmologia da Faculdade de Cièncias Médicas da Santa Casa de São Paulo - Rua Dr. Cesário Mota Junior, 112 - 01221 - São Paulo, SP - Brasil.

**** Do Serviço de Nutrição do Instituto de Saúde da Secretaria de Estado da Saúde do Estado de São Paulo - Rua São Luiz, 99 - São Paulo, SP - Brasill. 
WILson, D. et al. Arriboflavinose clínica em pré-escolares. filhos de migrantes em trânsito pela Central de Triagem e Encaminhamento, na Capital do Estado de São Paulo, Brasil. Rev. Saúde públ., S. Paulo, 12:351-5, 1978.

zaram vários tipos de inquéritos nutricionais, submeteram-se os pré-escolares também a exames clínico e oftalmológico. Nossa surpresa foi grande ao examinarmos as fichas clínicas, por constatar que a prevalência de sinais de arriboflavinose superava de muito aquela encontrada em estudos anteriores. Feita a constatação, nos foi impossivel introduzir o exame bioquímico, pois o trabalho já estava em fase adiantada e o número de dosagens seria decididamente insatisfatório.

Contentamo-nos, portanto, com o exame clínico, que servirá como brado de alerta para aqueles que, futuramente, venham a fazer pesquisas sobre estado nutricional, assim como às autoridades envolvidas com o problema da desnutrição entre nós.

\section{METODOLOGIA}

Foran examinados clinicamente todos os pré-escolares (109), de 2 a 7 anos de idade, filhos de migrantes em trânsito pela CETREN, durante o mês de janeiro de 1978, procedentes de várias regiões do Brasil. $O$ interesse da pesquisa evidenciou não ser necessário subdividi-los segundo as regiões de onde procediam, pois era meta estabelecer o estado nutricional do migrante nacional, qualquer que fosse sua origem.

Foram pesquisados os seguintes sinais clinicos atribuiveis à arriboflavinose ${ }^{1}$ :

Seborréia: nasolabial
nasal
frontal
retroauricular
Lesão do vestibulo nasal
Lesões das bordas palpebrais
Blefarite angular
Blefarite
Injeção circuncorneal
Estomatite angular
Queilose
Língua magenta

\section{RESULTADOS}

\section{Apresentação e discussão}

Sinais clinicos mais freqüentes

Pela Tabela 1 podemos ver quais is sinais clinicos que mais frequientemente apareceram. Podemos notar a alta prevalência de blefarite, lesão do vestibulo nasal e queilose. A injeção circuncorneal apresentou-se em apenas $7 \%$ dos examinados e, ao contrário do que se tem feito no passado, o exame foi realizado com um sistema óptico de ampliação.

As lesōes mais freqüentes neste estudo diferem daquelas encontradas em outros, mas ainda não se achou uma explicação para essa variabilidade, que se faz presente não apenas em nossas pesquisas ${ }^{3}$, mas também em trabalhos experimentais 1 . Cumpre-nos, por ora, apenas registrar () fato.

Freqüencia de pessoas apresentando sinais clinicos

$\mathrm{Na}$ Tabela 2 podemos apreciar o número de pessoas apresentando sinais clínicos de arriboflavinose. Podemos ver que $31 \%$ dos examinados apresentaram apenas um sinal de carência, enquanto que apenas $1 \%$ apresentou 4 sinais clínicos ao mesmo tempo. Tivemos ocasião, em trabalho anterior : de discutir o diagnóstico baseado em numero de sinais clínicos apresentados pelos pacientes. Se fossemos adotar o critério clínico, isto é, três ou mais sinais, para diagnosticar arriboflavinose, teríamos $15 \%$ dos examinados carentes de riboflavina. Este número supera acentuadamente aqueles encontrados em localidades do Estado de São Paulo ${ }^{3}$, que variaram entre $0,4 \%$ e $3,8 \%$, en média, $0,9 \%$. Este fato, tomado isoladamente, já indica uma situação extremamente grave enı termos de problema de saúde pública. Se adotarmos, entretanto, o critério que considera positivos aqueles individuos que apresentam pelo menos um sinal clínico de arriboflavinose, veremos que $68 \%$ das crianças examinadas, nele se enquadram 
WILSON, D. et al, Arriboflavinose clínica em p ré-escolares, filhos de migrantes em trânsito pela Central de Triagem e Encaminhamento, na Capital do Estado de São Paulo, Brasil. Rev. Saúde públ., S. Paulo, 12:351-5, 1978.

T A B E L A 1

Sinais clínicos de arriboflavinose em 109 pré-escolares, filhos de migrantes em trânsito pela CE'TREN, segun .o sua freqüência. São Paulo, 1977.

\begin{tabular}{l|c|c}
\hline \multicolumn{1}{c}{ Sinais clínicos } & No de pessoas & $\%$ \\
\hline Blefarite & 32 & 29 \\
Lesão do vestíbulo nasal & 29 & 27 \\
Queilose & 28 & 26 \\
Estomatite angular & 13 & 12 \\
Lesões das bordas palpebrais & 8 & 7 \\
Injeção circuncorneal & 8 & 7 \\
Blefarite angular & 5 & 5 \\
Língua magenta & 4 & 4 \\
Seborréia nasal & 2 & 2 \\
Seborréia retroauricular & 2 & 2 \\
\hline
\end{tabular}

T A B E L A 2

Freqüência dos 109 pré-escolares, filhos de migrantes em trânsito pela CETREN segundo o número de sinais clínicos apresentados. São Paulo, 1977.

\begin{tabular}{|c|c|c|c|c|}
\hline \multirow{2}{*}{$\mathrm{N}^{\circ}$ de sinais clínicos } & \multicolumn{2}{|c|}{ Freqüência de pré-escolares } & \multicolumn{2}{|c|}{ Freqüências acumuladas } \\
\hline & No & $\%$ & Ne & $\%$ \\
\hline 4 & 1 & 1 & 1 & 1 \\
\hline 3 & 15 & 14 & 16 & 15 \\
\hline 2 & 24 & 22 & 40 & 37 \\
\hline 1 & 34 & 31 & 74 & 68 \\
\hline 0 & 35 & 32 & 109 & 100 \\
\hline Total & 109 & 100 & 109 & 100 \\
\hline
\end{tabular}

(Tabela 2). É certo que entre esses préescolares encontrar-se-ão muitos falso-positivos, mas o fato de $15 \%$ dos examinados apresentarem pelo menos 3 sinais clínicos leva-nos a crer que o número de falsopositivos deve ser muito pequeno.

Considerando, ainda, que o exame clínico nos mostra a doença apenas na sua fase mais avançada, a situação da carência neste grupo humano deve ser ainda mais grave (talvez toda a população amostral seja carente), embora não possamos quantificar a magnitude da carência bioquimica, isto é, a fase sub-clínica da arriboflavinose.
Cumpre-nos também lembrar que esta população difere em muito daquelas estudadas em trabalho anterior ${ }^{3}$, pois, são migrantes em trânsito, de baixo nível sócio-econômico e que apresentam maiores dificuldades para obterem uma alimentação adequada, nos quais já se demonstrou existir outros problemas carenciais, como a hipovitaminose $A^{2}$.

Não foi possivel quantificar a carência bioquímica, mas tudo leva a crer que tenha alta prevalência neste grupo. 
WILSON, D. et al. Arriboflavinose clínica em pré-escolares, filhos de migrantes em trânsito pela Central de Triagem e Encaminhamento, na Capital do Estado de São Paulo, Brasil. Rev. Saúde públ., S. Paulo, 12:351-5, 1978.

T A B E L A 3

Associações de dois sinais clínicos encontrados em 109 pré-escolares, filhos de migrantes em trânsito pela CETREN. São Paulo, 1977.

\begin{tabular}{l|cc}
\hline \multicolumn{1}{c|}{ Sinais clínicos } & $\begin{array}{c}\text { No de } \\
\text { pré-escolares }\end{array}$ & $\%$ \\
\hline Lesões das bordas palpebrais + injeção circuncorneal & 4 & 4 \\
Blefarite angular + blefarite & 4 & 4 \\
Lesão do vestíbulo nasal + queilose & 3 & 3 \\
Lesão do vestíbulo nasal + blefarite & 2 & 2 \\
Blefarite + estomatite angular & 2 & 2 \\
Blefarite + queilose & 2 & 2 \\
Injeção circuncorneal + queilose & 2 & 2 \\
Seborréia nasal + queilose & 1 & $\mathbf{1}$ \\
Lesão do vestíbulo nasal + língua magenta & 1 & 1 \\
Blefarite + injeção circuncorneal & 1 & 1 \\
Estomatite angular + queilose & 1 & $\mathbf{1}$ \\
Blefarite angular + queilose & 1 & $\mathbf{1}$ \\
\hline
\end{tabular}

T A B E L A 4

Associações de três sinais clínicos encontrados em 109 pré-escolares, filhos de migrantes, em trânsito pela CETREN. São Paulo, 1977.

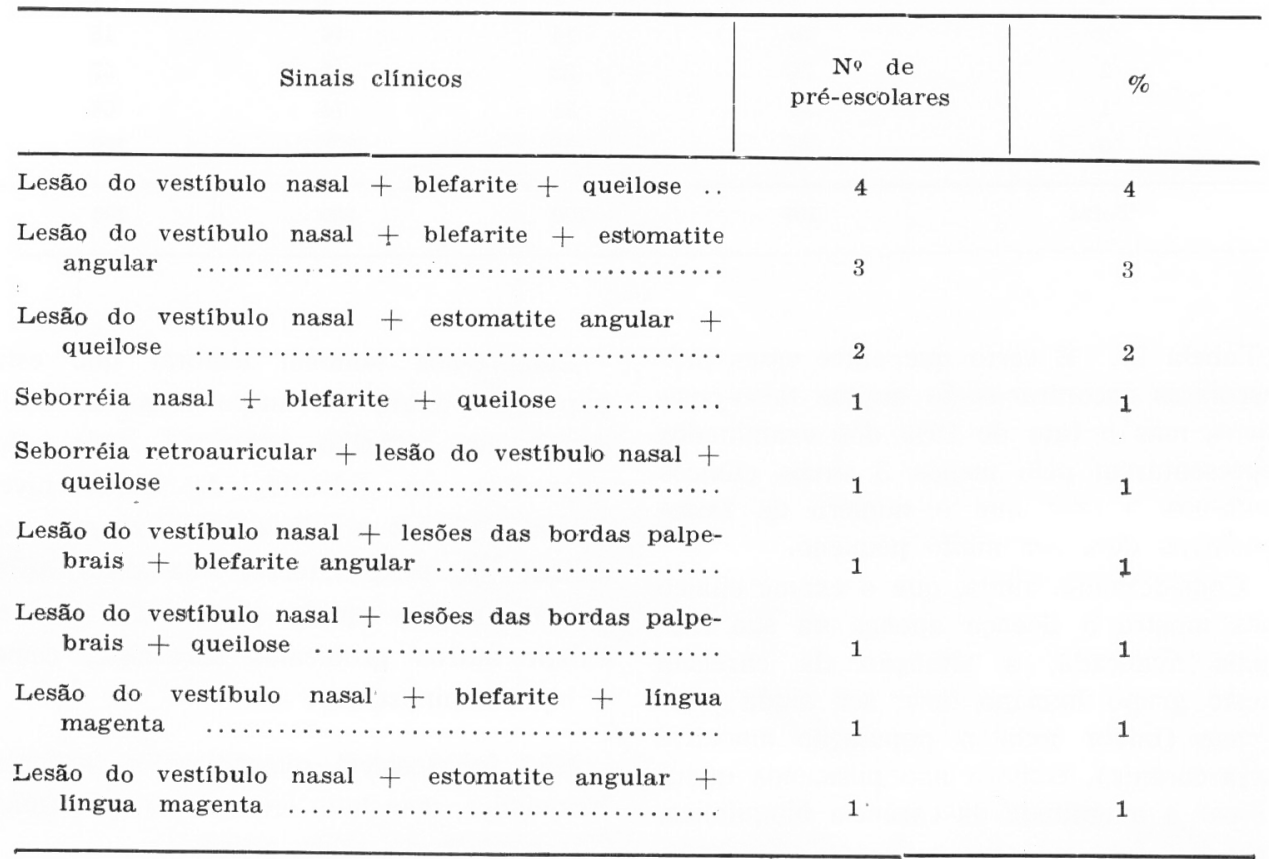


WILSON, D. et al. Arriboflavinose clínica em pré-escolares, filhos de migrantes em trânsito pela Central de Triagem e Encaminhamento, na Capital do Estado de São Paulo, Brasil. Rev. Saúde públ., S. Paulo, 12:351-5, 1978.

Associações de lesões

Houve grande variabilidade na associação de sinais clínicos. Podemos apreciar essas associações nas Tabelas 3 e 4 .

Houve ainda uma criança que apresentou quatro sinais clínicos, a saber: lesão do vestíbulo nasal, estomatite angular, queilose e língua magenta.

\section{CONCLUSOESS}

1. A arriboflavinose é sério problema de Saúde Pública nos pré-escolares filhos de migrantes em trânsito pela CETREN.
2. O nivel de carência é elevado, pois $68 \%$ das crianças apresentaram pelo menos um sinal clínico de arriboflavinose, $14 \%$ três sinais clínicos e $1 \%$ quatro sinais clínicos.

\section{Nota dos autores}

Como se tratasse de pessoas em trânsito, com permanência máxima de 72 horas na CETREN, a única medida factível foi a administração de riboflavina, realizada através da distribuição de cápsulas, num total de $200 \mathrm{mg}$ por criança examinada, em doses fracionadas.

WiLSON, D. et al. IClinical riboflavin deficiency in pre-school age children of migrants in transit through Central de Triagem $e$ Encaminhamento in the Capital of the State of S. Paulo, Brazil.] Rev. Saúde públ., S. Paulo, 12:351-5, 1978.

ABSTRACT: A clinical study was carried out on 109 pre-school age children of migrants in transit through Central de Triagem e Encaminhamento (CETREN). A very high prevalence rate regarding arriboflavinosis was found. The only feasible measure was administration of riboflavin in capsules to all children examined.

UnITERms: Arriboflavinosis, pre-school children. Riboflavin.

\section{REFERENCIAS BIBLIOGRAFICAS}

1. NUNEZ DEL PRADO, J. W. Arriboflavinosis en el Hospital Obrero de ICA. Rev. Med. Caja nac. Seg. soc., Lima, 20: 313-413, 1971.

2. RONCADA, M. J. Inquérito entre migrantes atendidos pela Central de Triagem e Encaminhamento, na Capital do Estado de São Paulo, Brasil. II. Aspectos bioquímicos da hipovitamino- se A. Rev. Saúde públ., S. Paulo, 9: 313-29, 1975.

3. WILSON, D. et al. Estudo da riboflavina: exames bioquímico e clínico em comunidades do Estado de São Paulo, Brasil. Rev. Saúde públ., S. Paulo, 11:13-26, 1977.

Recebido para publicação em 09/09/197\% Aprovado para publicąão em 25/10/1977 